\title{
Применение модели нейроннъх сетей для поддержки принятия решения абитуриента по выбору специальности
}

T.M. Зубкова ${ }^{1}$, д.m.н., npoфpeccop, bars87@mail.ru,

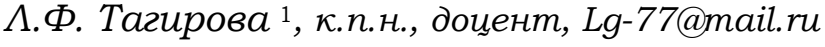

1 Оренбургский государственный университет, г. Оренбург, 460018, Россия

Одной из задач приемной комиссии является консультирование абитуриентов по имеющимся специальностям. После короткой беседы и анализа документов сотрудник может предложить специальности, на которые абитуриенту лучше всего подать заявление. Однако такая консультация зачастую носит субъективный характер.

В статье представлена разработанная автоматизированная информационная система, позволяющая на основе анкетирования абитуриента сформировать рекомендации при выборе специальности обучения. В качестве метода для выбора предлагается использовать нейронную сеть. Модель нейронной сети - многослойный перцептрон.

Перцептрон подлежит обучению, то есть требуется изменение значений синаптических весов. Нейросеть обучается на векторах данных лучших выпускников или студентов. Обучение нейросети происходит один раз перед началом работы приемной комиссии, после чего значения синаптических весов сохраняются для последующего использования. После предоставления абитуриентом документа об образовании и заполненной анкеты информация заносится в БД и инициируется запуск алгоритма классификации. Каждый выход нейросети соответствует отдельной специальности.

Нейросеть работает с аналоговыми данными. Для определения номера класса, к которому следует отнести входной вектор данных об абитуриенте, использован принцип наибольшего отклика, то есть наибольшее значение какого-либо из выходов соответствует распознанному классу. В качестве рекомендуемой специальности выбирается та, которой соответствует выход с наибольшим значением отклика.

Внедрение автоматизированной информационной системы позволит оказать абитуриенту интеллектуальную поддержку при принятии решения в профессиональном самоопределении, а также облегчить труд сотрудника приемной комиссии учебного заведения.

Ключевые слова: профессиональное самоопределение, искусственный интеллект, нейронные сети, многослойный периептрон, автоматизированная информачионная система.

Ежегодно во множестве образовательных учреждений работают приемные комиссии, одной из задач сотрудников которой является консультирование абитуриентов по имеющимся специальностям.

При формировании качественной рекомендации сотрудникам учреждения образования следует учитывать интеллектуальные способности абитуриента, которые отражаются в документе о базовом образовании, и его личностные качества, представление о которых можно получить путем психолого-педагогического анкетирования. Использование автоматизированной информационной системы (АИС) на этапе профессионального самоопределения абитуриента поможет повысить степень правильности принятого решения при выборе специальности обучения и оказать интеллектуальную поддержку на основе оценки интеллектуальных и личностных качеств.

\section{Методика решения поставленной задачи}

Проблемой профессионального самоопределения, а также построения систем поддержки принятия решений в различных областях занимались многие российские и зарубежные исследователи.

В работе [1] авторами предложено решение проблемы управления профессиональным самоопределением выпускника вуза на основе решающих деревьев. Рассмотрена потоковая модель функционирования вуза, выявлен характер функциональной зависимости пропускной способности вуза от профессионального самоопределения студента. На основе ответов респондентов на вопросы анкеты, содержащей количественную и качественную информацию, методами решающих деревьев построены модели трудоустройства выпускников всего вуза и каждого факультета в отдельности. Постро- 
енные деревья имеют различные корни в зависимости от факультетов и различные приоритеты значимости атрибутов. На их основе получена система продукционных правил, позволяющая формировать рекомендации по управлению траекторией профессионального самоопределения выпускников.

В статье [2] рассмотрена информационная система, которая автоматизирует профориентационные процедуры (просвещение, диагностика и консультирование) и предоставляет пользователям подробную информацию о современных профессиях, помогает реализовать более эффективную поддержку принятия решений и определить индивидуальные предпочтения на основе личностных данных.

Авторами [3] разработана программная система, использующая методы формирования перечня понятий и вычисление коэффициентов применения для определения предрасположенности к конкретным видам деятельности. В ходе работы системы осуществляется выбор подходящего направления подготовки или определение предрасположенности к конкретным видам деятельности на основании личных качеств, склонностей, которые выявляются на этапе тестирования.

Авторы статьи [4] представили результаты разработки новой компьютерной профориентационной методики для школьников старших классов на основе авторской «формулы профессии» и современной схемы анализа профессий. Они рассматривают преимущество трехуровневых оценок школьников («хочу», «смогу сейчас» и «смог бы в будущем») перед традиционным оцениванием школьником своих актуальных предпочтений. В процессе выбора профессии предпочитаемые характеристики будущей трудовой деятельности задаются не в виде прямых оценок их традиционных обозначений (предмет, цели, средства, условия и др.), а через парные сравнения основных трудовых и учебных действий.

Аналогичные подходы по принятию решений находят применение в разных сферах. Например, в [5] описано применение конкретных методов анализа принятия решений с использованием нескольких критериев для определения соответствующего поставщика автономного поезда в определенной производственно-распределительной компании. При определения подходящего компромиссного решения использованы методы WSA, скоринга, TOPSIS. Для применения каждого метода отдельные поставщики сортируются в зависимо- сти от пригодности для исследуемой компании на основе релевантности со всеми определенными критериями и их весами. Результаты, полученные отдельными методами, сравниваются друг с другом и определяется компромиссный поставщик автономной железнодорожной системы, которая должна быть реализована в выбранной компании.

В работе [6] описан новый тип системы поддержки принятия решений в чрезвычайных ситуациях, целью которой является улучшение процесса принятия решений руководителями в кризисных ситуациях путем их подключения к новым многочисленным источникам данных. Система оценивается с использованием реалистичного сценария кризиса с точки зрения пользовательских интерфейсов, ее способности интерпретировать данные в режиме реального времени и управлять большими данными. Авторы предлагают использовать его в качестве основы для будущих исследовательских работ по системам поддержки принятия решений, сталкивающимся со сложными меняющимися ситуациями.

Несмотря на достаточную изученность данного вопроса в теории и практике остается нерассмотренным вариант использования интеллектуальных методов для решения задачи профессионального выбора абитуриентов. Сегодня существует большое количество методов искусственного интеллекта, позволяющих решить данную задачу (экспертные системы, генетические алгоритмы, нечеткая логика, метод Байеса и т.д.).

Так как в рамках проводимого исследования выбора специальности абитуриентом имеется большое количество качественных параметров, было принято решение воспользоваться моделями нейронных сетей для создания ядра информационной системы. В качестве базовых источников теории нейронных сетей использовались материалы работ [7, 8] и результаты исследований современных ученых, посвященных использованию искусственных нейронных сетей [9], а также нейронных сетей в системах обработки данных [10].

Внедрение разработанной информационной системы поддержки принятия решения абитуриентом о выборе направления обучения позволит повысить степень верно принятого решения.

Задачу выбора специальности можно выразить в виде целевой функции

$$
P\left(l_{a}, k n_{a}, l_{g r}, k n_{g r}\right) \rightarrow \max ,
$$

где $P$ - степень принятия верного решения; $l_{a}$ личностные показатели абитуриента; $k n_{a}$ 
успеваемость абитуриента; $l_{g r}$ - личностные показатели лучших выпускников; $k n_{g r}$ - успеваемость лучших выпускников.

На рисунке 1 приведена диаграмма потоков данных при внедрении ПО поддержки принятия решения о выборе абитуриента в нотации DFD. Интеллектуальные показатели извлекаются из БД с информацией о студентах, их личных дел и переносятся в БД личностных и интеллектуальных показателей студентов сотрудником учебного заведения через ПО. На данном этапе под лучшими выпускниками понимаются студенты, защитившие выпускные квалификационные работы на отлично. Личностные показатели выпускников предполагается извлекать из БД студентов, которые были перенесены из БД приемной комиссии прошлых периодов.

В ходе работы с АИС сотрудник приемной комиссии принимает от абитуриента аттестат, анкету и переносит эти данные в программное средство, где они проходят предварительную обработку. Исходя из целевой функции (повышение степени верного выбора) и результатов системного анализа требуется получить обобщенный портрет лучшего выпускника по каждой специальности и отнести абитуриента к одному из этих портретов.

Учитывая наличие набора данных, на базе которых должна осуществляться классифика- ция без учета новых данных, использовались искусственные нейронные сети (ИНС) прямого распространения, а также метод обучения с учителем. Это обосновано тем, что характеристики абитуриентов не должны запоминаться ИНС в процессе классификации.

Для осуществления классификации был выбран многослойный перцептрон. Данный тип нейросетей архитектурно довольно прост и отлично подходит для решения задач распознавания. Структурная схема многослойного перцептрона представлена на рисунке 2.

Перцептрон представляет собой математическую модель нейронной сети, которая принимает на вход определенные значения - сигналы - и формирует отклик на них в виде выходных сигналов [8].

$$
\text { Выход каждого нейрона } y_{j}^{k}=f\left(\sum_{i=1}^{k^{k-1}} w_{i j}^{k} \cdot y_{i}^{k-1}\right) \text {, }
$$

где $k$ - номер слоя; $n^{k-1}-$ количество нейронов в $k$-м слое; $w_{i j}$ - синаптический вес, $j=\overline{1, n^{k}}$; $y_{j}^{0}=x_{j}$ - входные значения, $j=\overline{1, n^{0}}, n^{0}-$ количество входов; $f=f(x)=\frac{1}{1+e^{-k x}}-$ активационная функция (сигмоида); $x_{j}=l_{p} \cup k n_{q}-$ краткое математическое описание перцептрона ( $p=1, \ldots, n_{1}$ - количество ответов в анкете; $q=1, \ldots, n_{1}-$ количество оценок; $j=1, \ldots$,

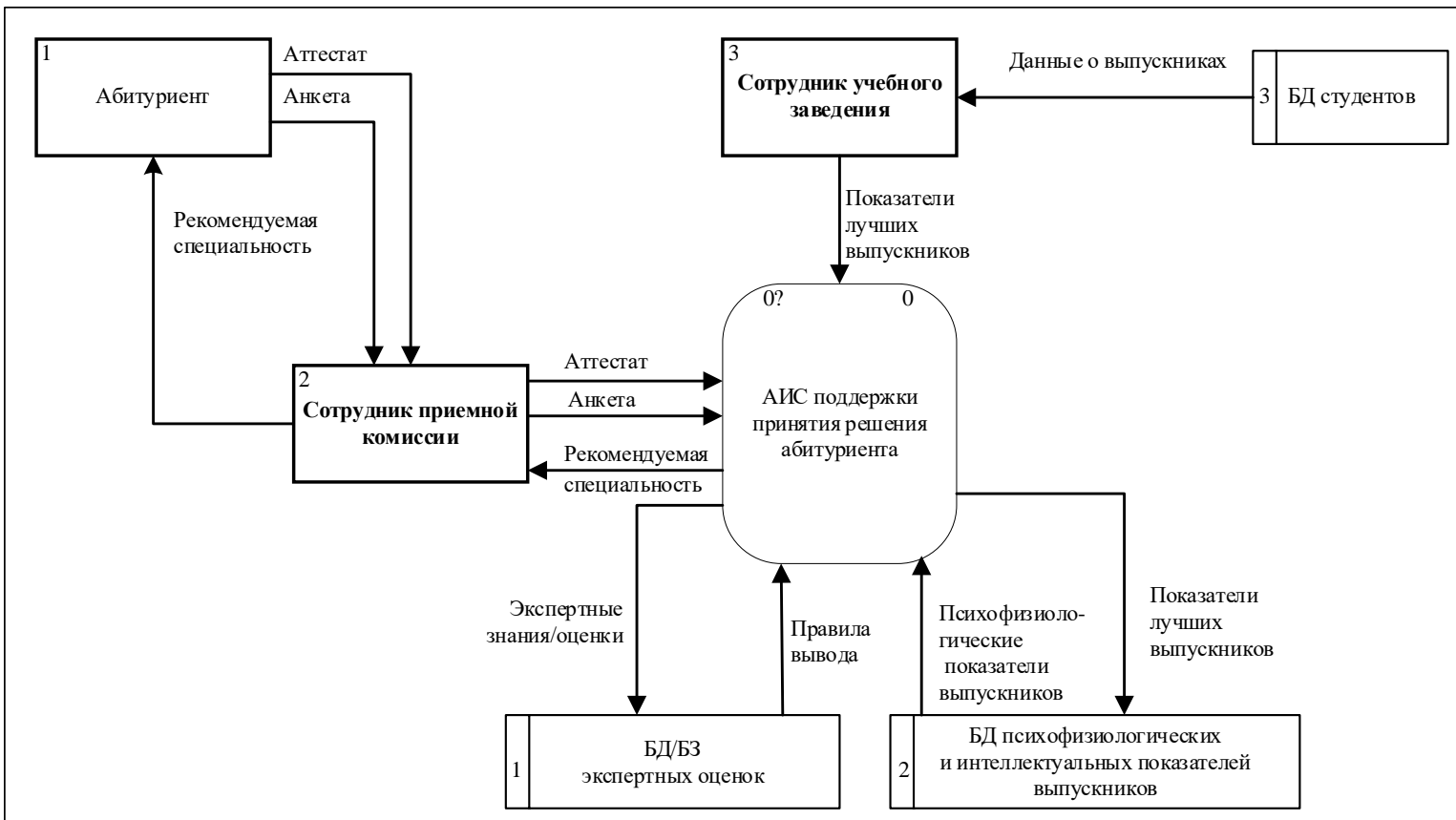

Рис. 1. Диаграмма потоков данных при реализащии прототипа поддержки принятия решения абитуриентом

Fig. 1. The diagram of data flows when implementing a prototype of decision support by an applicant 


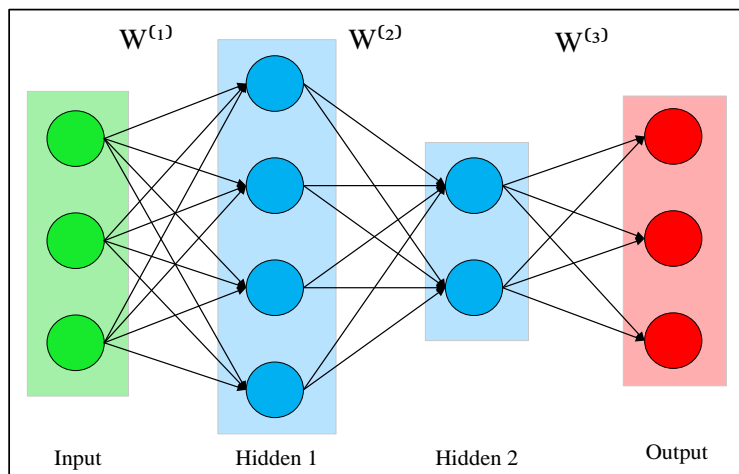

Рис. 2. Модель многослойного периеептрона

Fig. 2. The model of a multilayer perceptron

$\left(n_{1}+n_{2}\right)$ - общее количество входов; $l$ - ответы из анкеты; $k n$ - оценки абитуриента).

При этом на один и тот же набор входных значений с высокой долей вероятности формируется один и тот же отклик, то есть перцептрон запоминает образы входных значений и может их воспроизводить. Перцептрон состоит из нескольких слоев. Выделяют входной слой, один или несколько скрытых слоев и выходной слой. Под слоем понимается набор независимых друг от друга нейронов. Все нейроны разных слоев связаны друг с другом. Связь между двумя нейронами называют синапсом, или синаптической связью. Каждый нейрон имеет несколько входов и один выход. Каждый выход может быть входом для нескольких нейронов другого слоя. Как правило, все нейроны одного слоя связаны со всеми нейронами другого.

Каждый вход нейрона имеет коэффициент изменения этого входного значения - синаптический вес. Сам нейрон является сумматором входных значений, помноженных на значение синаптического веса, то есть происходит формирование средневзвешенной суммы. Так как значения средневзвешенной суммы могут иметь весьма большую дисперсию, их нормализуют, используя функцию активации или пороговую функцию, как правило, сигмоидальную функцию.

Перцептрон, как и любая другая нейронная сеть, подлежит обучению, то есть требуется изменение значений синаптических весов. Перцептрон относится к сетям, обучаемым с учителем: имеется набор входных значений (обучающая выборка) и для каждого входного значения задано ассоциированное с ним выходное значение. Обучение заключается в коррекции синаптических весов таким образом, чтобы сеть на входной набор выдавала нужное выходное значение.
Обучение - процесс довольно долгий, поэтому и были разработаны способы для его ускорения и повышения качества. Один из них - метод обратного распространения ошибки обучения, когда величина ошибки учитывается при коррекции синаптических весов. Эта величина рассчитывается от нейронов выходного слоя к нейронам входного слоя.

Таким образом, совокупность значений синаптических весов обученного перцептрона запоминает образы множества объектов и хранит в себе их свойства (причем заранее неизвестные) или скрытые закономерности. Это свойство можно использовать на практике [8].

Количество слоев и нейронов в каждом слое подбирается экспериментальным путем. В ходе проводимого исследования была подобрана следующая схема: 46-30-10-5, то есть во входном слое 46 нейронов, в двух скрытых слоях 30 и 10 нейронов соответственно, в выходном слое 5 нейронов. В данном случае варьировать можно только нейронами скрытых слоев, так как количество входов и выходов фиксированHoe.

Нейросеть будет работать с аналоговыми данными. Для определения номера класса, к которому следует отнести входной вектор данных об абитуриенте, воспользовались принципом наибольшего отклика, то есть наибольшее значение какого-либо из выходов соответствует распознанному классу. Для коррекции синаптических весов использовали алгоритм обратного распространения ошибки. Правило получения результата классификации: $\left\{y_{m}\right\}=$ $=f\left(x_{j}\right)$, где $m-$ количество выходов; $f-$ нейросеть; $A=\max \left(\left\{y_{m}\right\}\right), A-$ номер выхода с максимальным значением, то есть рекомендуемая специальность.

В таблице представлено правило получения результата классификации: переменные от $x_{1}$ до $x_{n 1}$ - оценки абитуриента, от $x_{n 1+1}$ до $x_{n 1+2}-$ ответы из анкеты абитуриента по определению личностных качеств, от $y_{1}$ до $y_{m}-$ выходы нейросети.

\section{Правило получения результата классификации}

The rule for obtaining the classification result

\begin{tabular}{|l|c|c|c|c|c|}
\hline Специальность & $\boldsymbol{x}_{\mathbf{1}}$ & $\boldsymbol{x}_{\mathbf{2}}$ & $\boldsymbol{x}_{\mathbf{3}}$ & $\ldots$ & $\boldsymbol{x}_{\boldsymbol{n} \mathbf{1 + n 2}}$ \\
\hline Специальность 1 & 4 & 5 & 3 & $\ldots$ & 7 \\
\hline Специальность 2 & 5 & 5 & 5 & $\ldots$ & 5 \\
\hline Специальность 3 & 3 & 5 & 4 & $\ldots$ & 10 \\
\hline Специальность 4 & 4 & 5 & 5 & $\ldots$ & 0 \\
\hline Специальность 5 & 5 & 4 & 3 & $\ldots$ & 8 \\
\hline
\end{tabular}


Входными данными нейросети являются векторы, объединяющие оценки (от 3 до 5 баллов, 16 значений) и ответы из анкеты (от 0 до 10 баллов, 30 вопросов-утверждений) выпускников или абитуриентов.

Нейросеть обучается на векторах данных лучших выпускников или студентов. Обучение нейросети происходит один раз перед началом работы приемной комиссии, и значения синаптических весов сохраняются для последующего использования. После предоставления абитуриентом документа об образовании и заполненной анкеты его данные заносятся в БД и инициируется запуск алгоритма классификации. Каждый выход нейросети соответствует отдельной специальности.
В качестве рекомендуемой специальности выбирается та, которой соответствует выход с наибольшим значением отклика. Рекомендация непосредственно сообщается абитуриенту для принятия к сведению при окончательном выборе специальности обучения.

\section{Алгоритмизация моделирующего аппарата}

Решение задачи сводится к двум шагам: обучение перцептрона и сохранение синаптических весов в памяти и непосредственно классификация абитуриента к одной из специальностей на основе сформированных синаптических весов. Процесс обучения нейросети представлен на рисунке 3 .

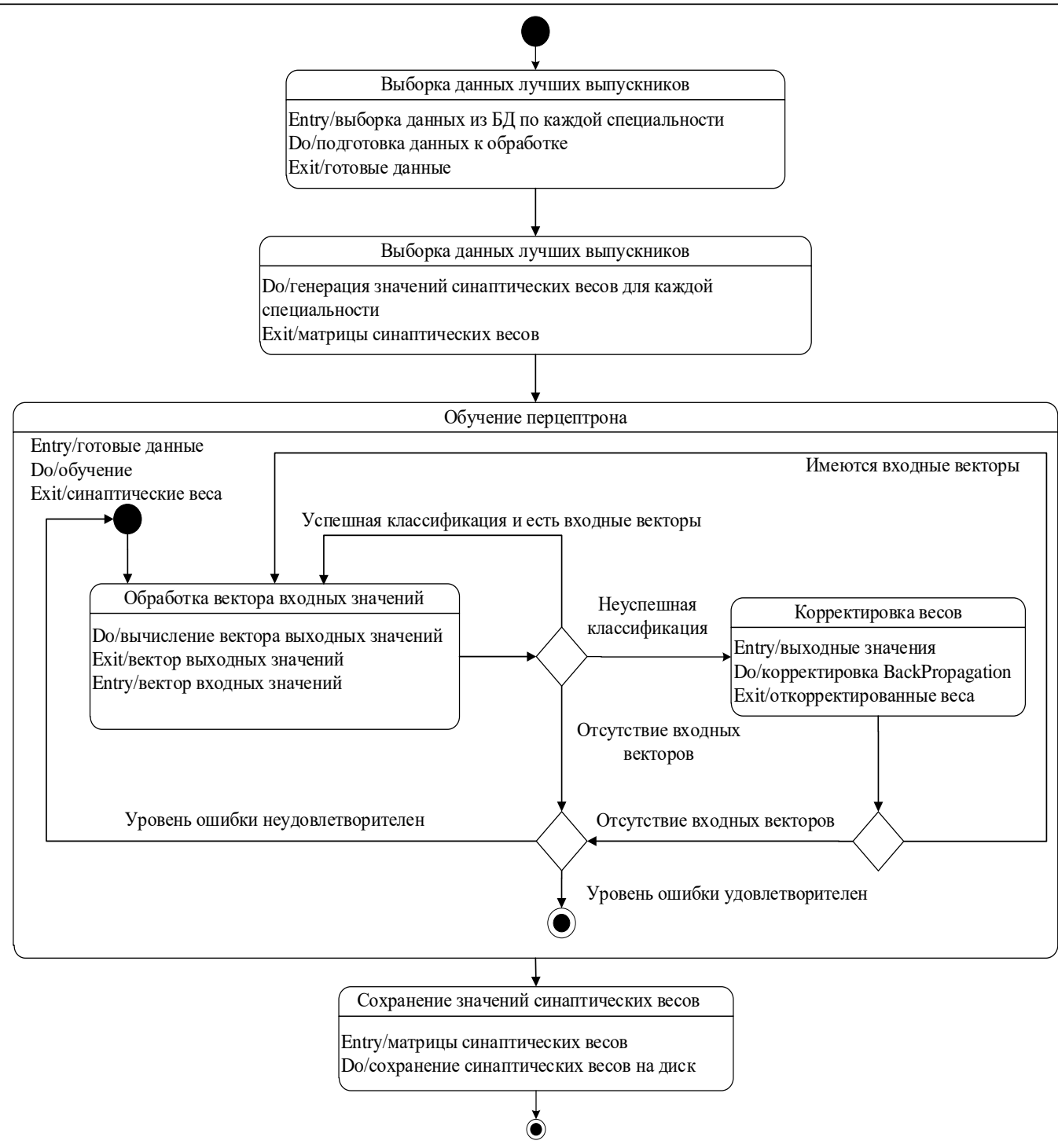

Рис. 3. Обучение периептрона

Fig. 3. The perceptron training 
Сначала происходит выборка данных лучших выпускников. Также можно включить данные студентов с положительной тенденцией в обучении. Выбранные данные предварительно структурируются - сохраняются в памяти в виде одномерных списков или массивов, так как данная структура удобна для подачи в нейросеть.

Далее происходят создание структуры нейросети в памяти, установка начальных значений синаптических весов и обучение перцептрона. В сеть подается входной вектор. Каждый входной вектор содержит также правильный ответ - основу для обучения. Затем осуществляется прогон вектора через сеть. Если ответ сети совпадает с требуемым правильным ответом, происходит подача следующего вектора, если нет - коррекция синаптических весов. Данный процесс над одним набором данных осуществляется несколько раз до тех пор, пока не будет достигнута требуемая точность классификации. Каждый прогон набора данных называют эпохой обучения. При этом перед каждой новой эпохой случай- ным образом рекомендуется изменять порядок следования входных векторов. Когда обучение завершено, нейросеть сохраняется в файл для последующего использования.

Процесс непосредственной классификации абитуриента с помощью обученного перцептрона представлен на рисунке 4. Он схож с процессом обучения. Сначала выбирается абитуриент и формируется вектор входных значений. Затем сохраненная нейросеть загружается в память. Далее входной вектор, описывающий абитуриента, подается в нейросеть. Вычисляется отклик сети, определяется номер входа, которому соответствует максимальное значение. По номеру входа определяется специальность.

На рисунке 5 представлена диаграмма вариантов использования.

Для дополнительного уточнения функционал можно представить в нотации UML-диаграммой вариантов использования, позволяющей наглядно увидеть, какую функцию реализует каждый из пользователей системы в процессе обработки данных.

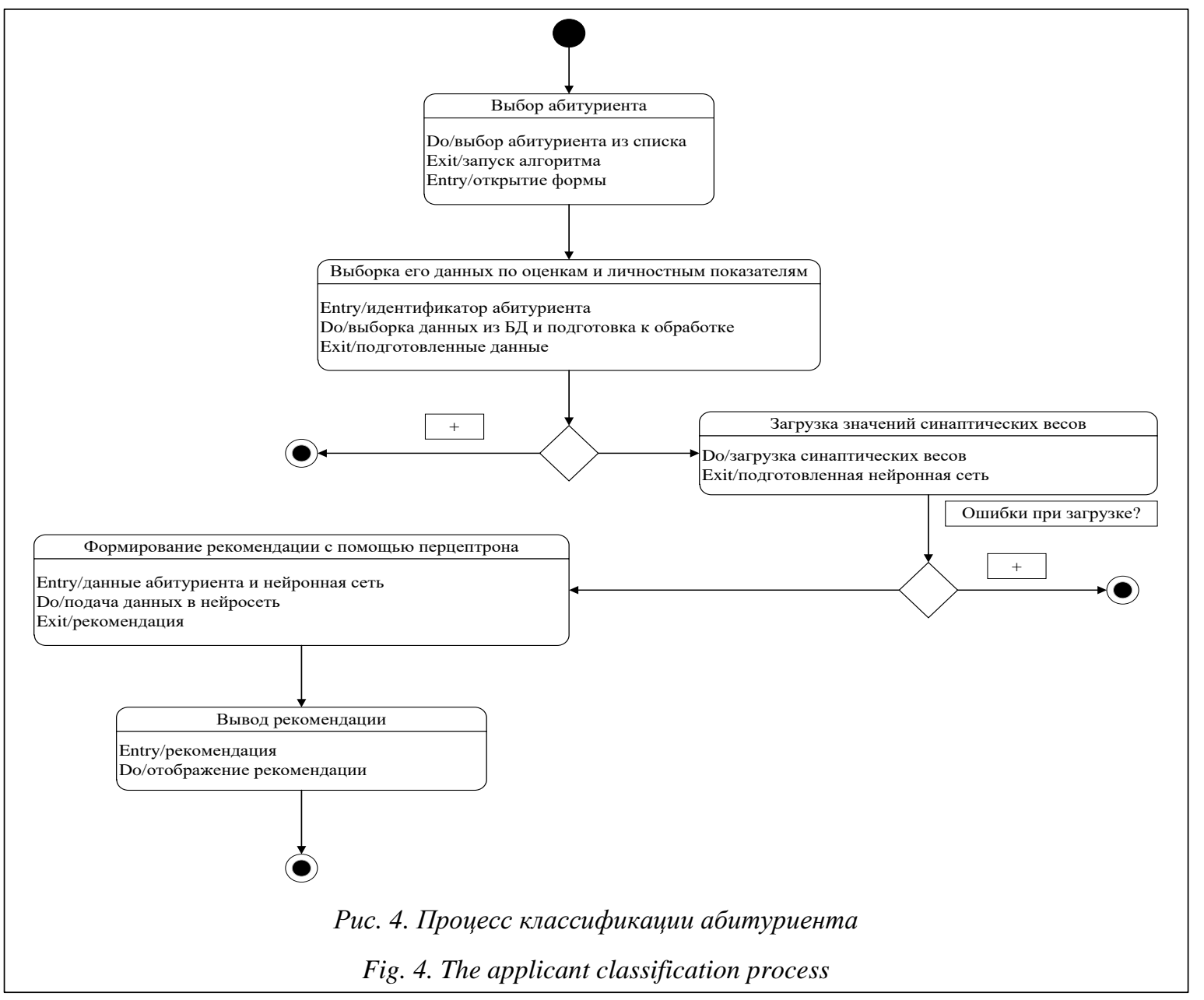




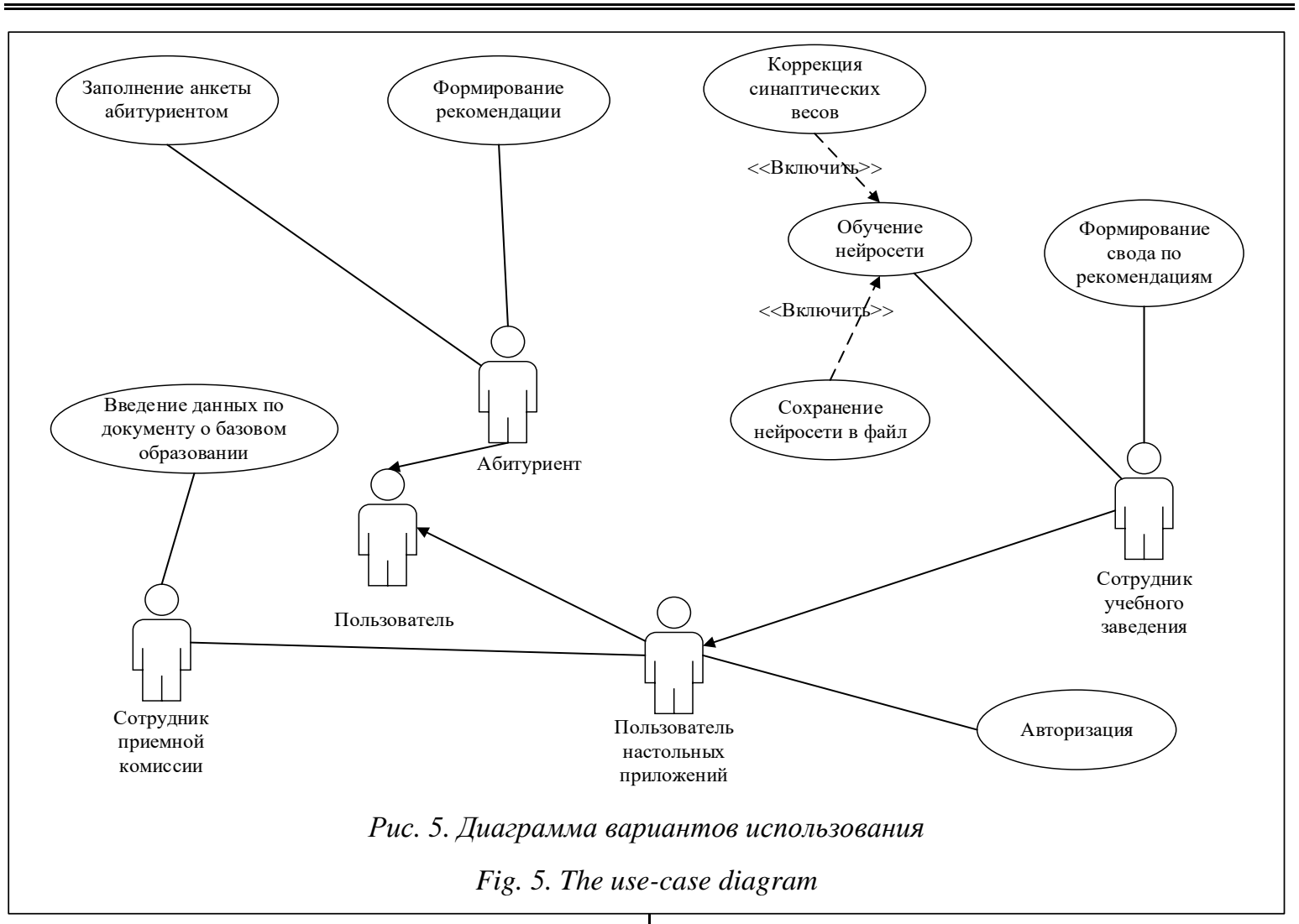

\section{Эксплуатация АИС}

Абитуриента консультируют по выбору специальности только по его запросу. Для этого его регистрируют в основной БД приемной комиссии. Далее происходит анкетирование (см. http://www.swsys.ru/uploaded/image/ 2021-2/2021-2-dop/8.jpg и http://www.swsys.ru/ uploaded/image/2021-2/2021-2-dop/9.jpg).

На данной странице указана краткая инструкция и перечислены утверждения анкеты. Всего утверждений 30 , на каждое из которых необходимо дать ответ в виде целого числа от нуля до десяти (см. http://www.swsys.ru/uploaded/image/2021-2/2021-2-dop/10.jpg).

После отправки анкеты происходит переход на страницу с резюме ответа (cм. http://www. swsys.ru/uploaded/image/2021-2/2021-2-dop/11.jpg).

На следующей странице будут отображены рекомендуемая специальность обучения и в роли справочной информации перечень всех доступных специальностей (см. http://www. swsys.ru/uploaded/image/2021-2/2021-2-dop/12.jpg).

Окно «Обучение нейросети» состоит из двух частей: слева расположены элементы управления для настройки параметров обучения и осуществления дополнительных операций, справа - текстовое поле для отображения сообщений, выводимых скриптом обучения нейросети. Для обучения нейросети нужно выбрать два основных параметра: источник обучающей выборки и режим обучения. Источником обучающих векторов может быть текстовый файл, где значения разделяются символом табуляции, или БД «Студент». Выбор источников обучающих векторов осуществляется флажками. Если выбрать и текстовый файл, и загрузку из БД, произойдет объединение двух наборов данных. Перед обучением выборку можно просмотреть (см. http://www.swsys.ru/ uploaded/image/2021-2/2021-2-dop/13.jpg).

Каждый сформированный набор сохраняется в текстовый файл и хранится до очередного формирования обучающей выборки.

Режим завершения обучения доступен с фиксированным количеством эпох обучения (cм. http://www.swsys.ru/uploaded/image/20212/2021-2-dop/26.jpg).

После каждого запуска обучения выведенные логи сохраняются в текстовый файл, и их можно просмотреть дополнительно.

После обучения синаптические веса нейросети сохраняются на диск в виде файла формата нейросетевой библиотеки для последующей загрузки в память и использования ее для классификации абитуриентов.

Для контроля работы нейросети можно воспользоваться соответствующим окном, вызы- 
ваемым из окна по работе с нейросетью (см. http://www.swsys.ru/uploaded/image/2021-2/ 2021-2-dop/14.jpg).

Положительная динамика в обучении студентов на текущий момент по доступным данным отмечается примерно у $74 \%$ студентов (около 3/4), что говорит об эффективности предложенного метода (см. http://www.swsys. ru/uploaded/image/2021-2/2021-2-dop/15.jpg).

\section{Заключение}

В данной статье для поддержки принятия решений были использованы искусственные нейронные сети, а для осуществления классификации выбран многослойный перцептрон.

Использование предложенной АИС позволит оказать интеллектуальную поддержку при принятии решения абитуриентом о выборе наиболее подходящей специальности на основе оценки интеллектуальных и личностных качеств, сократит время на обработку данных и повысит степень правильности принятых решений. При соответствующем заполнении БД АИС может быть адаптирована и для других задач по выбору принятия решений.

\section{Лuтература}

1. Болодурина И.П., Ханжина Н.В. Управление профессиональным самоопределением при трудоустройстве выпускника вуза на основе решающих деревьев // Интеллект. Инновации. Инвестиции. 2015. № 1. С. 15-22.

2. Похорукова М.Ю. Информационная поддержка принятия решений в процессе выбора профессии // Инновации и инвестиции. 2017. № 11. С. 59-62.

3. Путивцева Н.П., Зайцева Т.В., Пусная О.П., Игрунова С.В., Нестерова Е.В., Калюжная Е.В., Шуваева Е.Ю. О разработке автоматизированной системы выбора направления будущей профессиональной деятельности // Научные ведомости БелГУ. Сер.: Экономика. Информатика. 2016. № 16. C. $138-146$.

4. Гусев А.Н., Пряжников Н.С., Тюрин К.Г. Экспертная система профориентации школьников «Выбирай и поступай» // Вестн. Московского ун-та: Психология. 2016. № 4. С. 55-60.

5. Stopka O., Stopková M., L’upták V., Krile S. Application of the chosen multi-criteria decision-making methods to identify the autonomous train system supplier. Transport Problems, 2020, vol. 15, no. 2, pp. 45-57. DOI: $10.21307 /$ tp-2020-019.

6. Fertier A., Montarnal A., Truptil S., Bénaben F. A new emergency decision support system: The automatic interpretation and contextualisation of events to model a crisis situation in real-time. Decision Support Systems, 2020, vol. 133, pp. 113-260. DOI: 10.1016/j.dss.2020.113260.

7. Хайкин С. Нейронные сети. Полный курс; [пер. с англ.]. М.: Вильямс, 2006. 1104 с.

8. Рутковская Д., Пилиньский М., Рутковский Л. Нейронные сети, генетические алгоритмы и нечеткие системы. М.: Горячая линия-Телеком, 2006. 452 с.

9. Соловьева Т.А. Искусственные нейронные сети // Вестн. научн. конф. 2018. № 12-1. С. $105-106$.

10. Шилин Л.Ю., Навроцкий А.А., Стригалев Л.С. Нейронные сети в системах обработки данных // Big Data and Advanced Analytics. 2018. № 4. С. 392-394.

\section{Application of the neural network model to support acceptance decisions of the applicant by choice of specialty}

T.M. Zubkova ${ }^{1}$, Dr.Sc. (Engineering), Professor, bars87@mail.ru

L.F. Tagirova ${ }^{1}$, Ph.D. (Education), Associate Professor, LG-77@mail.ru

${ }^{1}$ Orenburg State University, Orenburg, 460018, Russian Federation

Abstract. The problem of the admissions office is to advise applicants in existing specialties upon admission. After a brief conversation and document analysis, the employee can offer specialties for which the applicant is best to apply. However, the consultation of an employee of the admissions committee is often subjective.

The paper presents the developed automated information system (AIS), which allows, based on the questionnaire of the applicant, to form recommendations when choosing a major. Using the neural networks is proposed as a method for selection. Proposed is a model of neural network - multilayer perceptron. 
Perceptron is to be trained, i.e. a change in synaptic weights is required. We trained the neural network on data vectors of the best graduates or students. Neural network training occurs once before the start of the admissions office, after which the values of the synaptic scales are preserved for subsequent use. After the applicant submits the education document and the completed questionnaire, information is entered into the database (DB) and the classification algorithm is started. Each output of the neural network corresponds to a separate specialty.

The neural network works with analog data. To determine the class number to which the input vector of the applicant data should be assigned, they used the principle of greatest response, that is, the largest value of the outputs corresponds to the recognized class. The recommended specialty is the one that corresponds to the output with the highest response value.

Introducing the AIS will allow the applicant to provide intellectual support when deciding on professional self-determination, as well as facilitate the employee's labor of the education institution admissions office.

Keywords: professional self-determination, artificial intelligence, neural networks, multilayer perceptron, automated information system.

\section{References}

1. Bolodurina I.P., Khanzhina N.V. Management of professional self-determination in the employment of a graduate of a university based on decisive trees. Intellect. Innovation. Investments, 2015, no. 1, pp. 15-22 (in Russ.).

2. Pokhorukova M.Yu. Information support for decision-making in the process of choosing a profession. Innovation and Investment, 2017, no. 11, pp. 59-62. (in Russ.).

3. Putivtseva N.P., Zaytseva T.V., Pusnaya O.P., Igrunova S.V., Nesterova E.V., Kalyuzhnaya E.V., Shuvaeva E.Yu. On the development of the automated system selection directions future careers. Belgorod State Univ. Sci. Bull. Economics Information Technologies, 2016, no. 16, pp. 138-146 (in Russ.).

4. Gusev A.N., Pryazhnikov N.S., Tyurin K.G. Expert system of career guidance for schoolchildren "Choose and act". Moscow Univ. Psychology Bull. Psychology, 2016, no. 4, pp. 55-60 (in Russ.).

5. Stopka O., Stopková M., L'upták V., Krile S. Application of the chosen multi-criteria decision-making methods to identify the autonomous train system supplier. Transport Problems, 2020, vol. 15, no. 2, pp. 45-57. DOI: 10.21307/tp-2020-019.

6. Fertier A., Montarnal A., Truptil S., Bénaben F. A new emergency decision support system: The automatic interpretation and contextualisation of events to model a crisis situation in real-time. Decision Support Systems, 2020, vol. 133, pp. 113-260. DOI: 10.1016/j.dss.2020.113260.

7. Haikin S. Neural Networks. A Comprehensive Foundation. NJ, Upper Saddle River, Prentice Hall Publ., 1999, 973 p. (Russ. ed.: Moscow, 2006, 1104 p.).

8. Rutkovskaya D., Pilinsky M., Rutkovsky L. Neural Networks, Genetic Algorithms and Fuzzy Systems. Moscow, 2006, 452 p. (in Russ.).

9. Soloveva T.A. Artificial neural networks. Bull. of Scientific Conf., 2018, no. 12-1, pp. 105-106. (in Russ.).

10. Shilin L.Yu., Navrotsky A.A., Strigalev L.S. Neural networks in data processing systems. Big Data and Advanced Analytics, 2018, no. 4, pp. 392-394 (in Russ.).

\section{Для цитирования}

Зубкова Т.М., Тагирова А.Ф. Применение модеми нейронных сетей для поддержки принятия решения абитуриента по выбору специальности // Программные продукты и системы. 2021. T. 34. № 2. C. 365-373. DOI: 10.15827/0236-235X.134.365-373.

\section{For citation}

Zubkova T.M., Tagirova L.F. Application of the neural network model to support acceptance decisions of the applicant by choice of specialty. Software \& Systems, 2021, vol. 34, no. 2, pp. 365-373 (in Russ.). DOI: 10.15827/0236-235X.134.365-373. 